ARTICLE

\title{
Piezoelectric domain walls in van der Waals antiferroelectric $\mathrm{CulnP}_{2} \mathrm{Se}_{6}$
}

Andrius Dziaugys ${ }^{1,13}$, Kyle Kelley (2,13, John A. Brehm³ ${ }^{3}$ Lei Tao 3,4, Alexander Puretzky², Tianli Feng 2,3, Andrew O'Hara (1) ${ }^{3}$, Sabine Neumayer (10 ${ }^{2}$, Marius Chyasnavichyus ${ }^{2}$, Eugene A. Eliseev (1) ${ }^{5}$, Juras Banys ${ }^{1}$, Yulian Vysochanskii id 6 ${ }^{6}$, Feng Ye (10 ${ }^{7}$, Bryan C. Chakoumakos ${ }^{7}$, Michael A. Susner ${ }^{8,9}$, Michael A. McGuire ${ }^{10}$, Sergei V. Kalinin (1D 2, Panchapakesan Ganesh (10) 2, Nina Balke (1) 2, Sokrates T. Pantelides (1) 3,11, Anna N. Morozovska ${ }^{12} \&$ Petro Maksymovych (i) ${ }^{2 \otimes}$

Polar van der Waals chalcogenophosphates exhibit unique properties, such as negative electrostriction and multi-well ferrielectricity, and enable combining dielectric and 2D electronic materials. Using low temperature piezoresponse force microscopy, we revealed coexistence of piezoelectric and non-piezoelectric phases in Culn $\mathrm{P}_{2} \mathrm{Se}_{6}$, forming unusual domain walls with enhanced piezoelectric response. From systematic imaging experiments we have inferred the formation of a partially polarized antiferroelectric state, with inclusions of structurally distinct ferrielectric domains enclosed by the corresponding phase boundaries. The assignment is strongly supported by optical spectroscopies and density-functionaltheory calculations. Enhanced piezoresponse at the ferrielectric/antiferroelectric phase boundary and the ability to manipulate this entity with electric field on the nanoscale expand the existing phenomenology of functional domain walls. At the same time, phase-coexistence in chalcogenophosphates may lead to rational strategies for incorporation of ferroic functionality into van der Waals heterostructures, with stronger resilience toward detrimental size-effects.

\footnotetext{
${ }^{1}$ Faculty of Physics, Vilnius University, Vilnius LT-01513, Lithuania. ${ }^{2}$ The Center for Nanophase Materials Sciences, Oak Ridge National Laboratory, Oak Ridge, TN 37831, USA. ${ }^{3}$ Department of Physics and Astronomy and Department of Electrical Engineering and Computer Science, Vanderbilt University, Nashville, TN 37235, USA. ${ }^{4}$ University of Chinese Academy of Sciences \& Institute of Physics, Chinese Academy of Sciences, Beijing, China. ${ }^{5}$ Institute for Problems of Materials Science, National Academy of Sciences of Ukraine, Krjijanovskogo 3, 03142 Kyiv, Ukraine. ${ }^{6}$ Institute of Solid State Physics and Chemistry, Uzhgorod University, 88000 Uzhgorod, Ukraine. ${ }^{7}$ Neutron Scattering Division, Oak Ridge National Laboratory, Oak Ridge, TN, USA. ${ }^{8}$ Materials and Manufacturing Directorate, Air Force Research Laboratory, WPAFB, OH 45433, USA. ${ }^{9}$ UES, Inc. 4401 Dayton-Xenia Rd., Dayton, OH 45432, USA. ${ }^{10}$ Materials Science and Technology Division, Oak Ridge National Laboratory, Oak Ridge, TN 37831, USA. ${ }^{11}$ Department of Electrical Engineering and Computer Science, Vanderbilt University, Nashville, TN 37235, USA. ${ }^{12}$ Institute of Physics, National Academy of Sciences of Ukraine, Prospect Nauky 46, Kyiv-28 03680, Ukraine. ${ }^{13}$ These authors contributed equally: Andrius Dziaugys, Kyle Kelley. ${ }^{凶}$ email: maksymovychp@ornl.gov
} 
ayered thiophosphates, with a general composition of $\mathrm{CuInP}_{2} Q_{6}(Q=\mathrm{S}, \mathrm{Se})^{1}$, have recently gained attention as candidate materials for two-dimensional ${ }^{2-4}$ (2D) or fewlayered ferroelectrics 2,5 . The sulfur ${ }^{6,7}$ and selenium ${ }^{8-10}$ compounds have similar structure of individual layers and a concomitant ferrielectric (FE) ordering, with $\mathrm{Cu}^{+}$and $\mathrm{In}^{3+}$ ions counter-displaced within individual layers, against the backbone of $\mathrm{P}_{2} \mathrm{Q}^{4-}{ }_{6}$ anions. The spontaneous polarization of the sulfide can range from $\sim 5 \mu \mathrm{C} / \mathrm{cm}^{2}$ to $\sim 12 \mu \mathrm{C} / \mathrm{cm}^{11}$ vs $\sim 2.5 \mu \mathrm{C} / \mathrm{cm}^{2}{ }^{12}$ in the selenide, in part due to larger off-centric $\mathrm{Cu}$ displacement in the sulfide. Despite the structural similarity, the reported properties of their phase transitions are quite different ${ }^{10}$. Other than the difference in the transition temperatures $\left(\sim 230 \mathrm{~K}^{8,9}\right.$ in the selenide vs $\sim 305 \mathrm{~K}$ in the sulfide $\left.{ }^{6,10}\right), \mathrm{CuInP}_{2} \mathrm{Se}_{6}$ exhibits a broader transition window compared to $\mathrm{CuInP}_{2} \mathrm{~S}_{6}$, as evidenced by macroscopic dielectric, caloric and thermal characterization ${ }^{9,10}$. It was proposed that this anomaly is evidence for the coexistence of ferrielectric and antiferroelectric (AFE) ordering, and an incommensurate phase that precedes ferroelectric ordering ${ }^{9}$. The properties of the intermixed S-Se compound are even more interesting, possibly involving a Lifshitz transition as well as polar glassy phases ${ }^{13}$. The apparent compatibility of chalcogenophosphates with a variety of polar orderings signifies comparatively weak dipolar correlations in the lattice. This property may be particularly pertinent toward prospective application of these materials as functional components of van der Waals heterostructures.

Indeed, recently, Song et al. ${ }^{12}$ proposed that ultrathin films of $\mathrm{CuInP}_{2} \mathrm{Se}_{6}$ develop an antiferroelectric ground state, with the ferrielectric-antiferroelectric crossover occurring at a thickness of $\sim 6-8$ layers. The primary driving force for the crossover is the depolarizing field that favors the antiferroelectric with net zero polarization. This feature is in contrast to perovskite oxides, such as the canonical $\mathrm{BaTiO}_{3}$, which become non-polar in the ultrathin limit ${ }^{14}$. However, at present, most nanoscale polar properties in both $\mathrm{CuInP}_{2} \mathrm{~S}_{6}$ and $\mathrm{CuInP}_{2} \mathrm{Se}_{6}$ remain to be understood, with respect to the mechanisms that screen spontaneous polarization at the interfaces, polarization switching, and the structure of the domains and their domain walls ${ }^{1}$ as well as the scalability down to the single-layer limit. Understanding these behaviors will also help to identify the possible mechanisms by which these materials can be functional in van der Waals heterostructures.

Here, we report the structure of polarization domains in bulk $\mathrm{CuInP}_{2} \mathrm{Se}_{6}$ utilizing quantitative imaging of nanoscale piezoelectric properties. Contrary to the expectation of ferrielectric ordering analogous to $\mathrm{CuInP}_{2} \mathrm{~S}_{6}$, we reveal a fundamentally different domain structure, with two markedly different values of piezoresponse. Moreover, the domain boundaries exhibit the strongest piezoelectric response, with up to fourfold enhancement compared to domain surfaces. CuInP $\mathrm{P}_{2} \mathrm{~S}_{6}$, on the other hand, features an expected domain structure, with nearly uniform value of piezoresponse within domains, alternating polarization orientation and domain walls with vanishing piezoresponse ${ }^{15-17}$. Despite the more complex polar structure of $\mathrm{CuInP}_{2} \mathrm{Se}_{6}$, the domains can be flexibly manipulated with applied fields. We explain the phenomena observed in $\mathrm{CuInP}_{2} \mathrm{Se}_{6}$ by considering the real-space domain structure of an antiferroelectric that is partially polarized in finite electric field. Our arguments are supported by Raman spectroscopy and DFT calculations indicating the possible coexistence of ferrielectric and antiferroelectric states. FE/AFE coexistence presents an intriguing opportunity for few-unit-cell thiophosphates, particularly within van der Waals heterostructures. At the same time, piezoelectric domain walls, which are polar phase boundaries in this case and can be readily manipulated by applied electric fields, present a new functional element for the domain-wall electronics paradigm ${ }^{18,19}$.

\section{Results and discussion}

Piezoresponse force microscopy does not exhibit any significant signal on the surface of $\mathrm{CuInP}_{2} \mathrm{Se}_{6}$ at room temperature in the paraelectric state, as expected. On a crystal cooled below $200 \mathrm{~K}$, the piezoresponse phase and amplitude images (Fig. 1a, b) reveal domains of various forms and sizes (the measured response is defined by the normal components of the piezoelectric coefficient $d_{3 j}=2 \varepsilon_{0} \varepsilon_{33} Q_{j 3} P_{3}$, where the $Q_{j 3}$ are components of electrostriction tensor, $P_{3}$ is the normal polarization component, and $\varepsilon_{33}$ is the normal component of the dielectric constant). Despite structural similarity, however, there is a stark contrast between $\mathrm{CuInP} \mathrm{P}_{2} \mathrm{Se}_{6}$ and $\mathrm{Cu}_{0.4} \mathrm{In}_{1.2} \mathrm{P}_{2} \mathrm{~S}_{6}$ as shown in Fig. 1. In $\mathrm{Cu}_{0.4} \mathrm{In}_{1.2} \mathrm{P}_{2} \mathrm{~S}_{6}$, the contrast of the piezoelectric signal.

comprises the non-polar dielectric phase $\operatorname{In}_{4 / 3} \mathrm{P}_{2} \mathrm{~S}_{6}$, with negligible piezoresponse, and the ferrielectric phase $\mathrm{CuInP}_{2} \mathrm{~S}_{6}$, whose piezoresponse is comparable to a ferroelectric: domains of opposite polarization have opposite signs of piezoresponse, with nearly zero signal at the domain wall in between (Fig. 1b, d) ${ }^{20,21}$. Cancellation of piezoresponse at the domain wall is a mutual consequence of nearly zero polarization at the wall, and possibly the mechanical cancellation effect due to opposite direction of surface deformation in the adjacent domains separated by the domain wall.

In $\mathrm{CuInP}_{2} \mathrm{Se}_{6}$, extended regions of negligible piezoresponse and extended regions of uniform piezoresponse also exist. However, in contrast to $\mathrm{Cu}_{0.4} \mathrm{In}_{1.2} \mathrm{P}_{2} \mathrm{~S}_{6}$, $\mathrm{CuInP}_{2} \mathrm{Se}_{6}$ crystals studied here are nearly stoichiometric, excluding the existence of a nonferroelectric phase, such as $\operatorname{In}_{4 / 3} \mathrm{P}_{2} \mathrm{~S}_{6}$ in $\mathrm{Cu}_{0.4} \mathrm{In}_{1.2} \mathrm{P}_{2} \mathrm{~S}_{6}$. Moreover, the regions of finite piezoresponse appear only below the transition and can be flexibly manipulated with applied fields (shown below in Fig. 2j). At the same time, the piezoresponse signal across a boundary separating the two distinct regions in $\mathrm{CuInP}_{2} \mathrm{Se}_{6}$ has maximum rather than minimum piezoresponse signal. These observations imply that $\mathrm{CuInP}_{2} \mathrm{Se}_{6}$ below the ferroic transition intrinsically exhibits at least two distinct structural phases. The imaging results are reproduced for a variety of tips, cleaved surfaces and are also observed irrespective of the underlying topography (Supplementary Fig. 1). By using PFM in ultrahigh vacuum on freshly cleaved and nearly atomically flat surfaces, as well as invoking the band-excitation methodology for piezoresponse $^{22}$, we have further ruled out simple experimental artifacts such as the underlying topography ${ }^{23}$, electrochemistry ${ }^{24}$, or mechanical properties of the contact ${ }^{25}$.

Further insight as well as confirmation of the above assignments is evidenced from the electric-field dependence of the measured piezoresponse (Fig. 2). It was previously pointed out that it is essential to check local electromechanical measurements for possible artefacts, such as the contribution of extrinsic electrostatics ${ }^{24}$. As seen in Fig. 2, the dominant effect of applied bias is to impose an offset on the measured signal in both $\mathrm{Cu}_{0.4} \mathrm{In}_{1.2} \mathrm{P}_{2} \mathrm{~S}_{6}$ and $\mathrm{CuInP}_{2} \mathrm{Se}_{6}$, while keeping the contrast between entities largely unchanged.

This conclusion is further confirmed by analysis of select $1 \mathrm{D}$ profiles from the data in Fig. 2, as shown in Fig. 3. Indeed, relative enhancement of piezoresponse is maintained irrespective of applied field (Fig. 3a, c), while domain walls in $\mathrm{Cu}_{0.4} \mathrm{In}_{1.2} \mathrm{P}_{2} \mathrm{~S}_{6}$ reveal approximately average signal between up and downoriented domains (Fig. 3b). At the same time, at both $-3 \mathrm{~V}$ (Fig. 2f) and $+2 \mathrm{~V}$ (Fig. 2j), the domain structure of $\mathrm{CuInP}_{2} \mathrm{Se}_{6}$ begins to evolve in applied field, respectively shrinking and growing the regions of finite piezoresponse.

Given all of the above measurements, we propose that $\mathrm{CuInP}_{2} \mathrm{Se}_{6}$ exhibits coexistence of antipolar and polar regions, identified, respectively, by zero and finite piezoresponse signals. The domain walls are then the boundaries separating these 
a
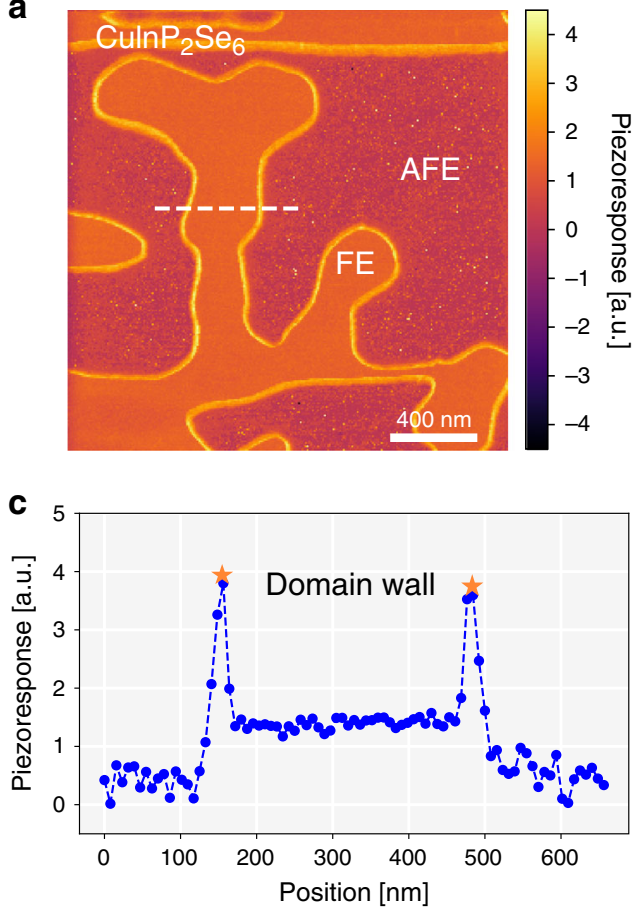

b

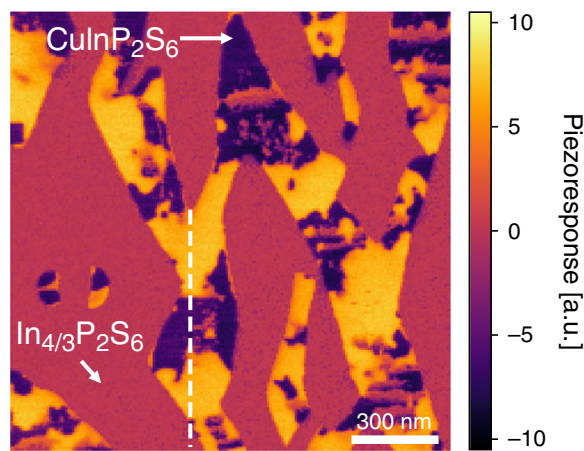

d

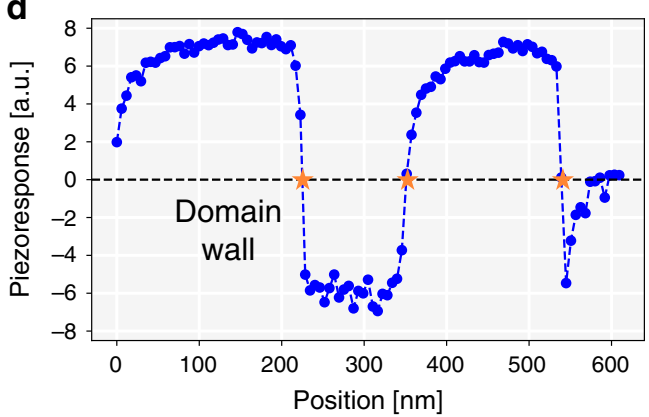

Fig. 1 Comparison of piezoresponse due to domains and domain walls in $\mathbf{C u I n} \mathbf{P}_{\mathbf{2}} \mathbf{S e} \mathbf{6}_{\mathbf{6}}$ and $\mathbf{C u}_{\mathbf{0 . 4}} \mathbf{I} \mathbf{n}_{\mathbf{1 . 2}} \mathbf{P}_{\mathbf{2}} \mathbf{S}_{\mathbf{6}}$. CulnP $\mathrm{Se}_{6}$ surfaces were measured at $140 \mathrm{~K}$ in ultrahigh vacuum and that of $\mathrm{CulnP}_{2} \mathrm{~S}_{6}$-at room temperature in controlled environment: $\mathbf{a}$, $\mathbf{b}$ piezoresponse $\operatorname{signals} A \cos (\theta)$ (where $A$ is the amplitude and $\theta$ the phase of piezoresponse)-revealing at least three distinct magnitudes of piezoresponse for both materials, and $\mathbf{c}$, $\mathbf{d}$ line-profiles of piezoresponse along the white dashed lines, crossing domain boundaries in $\mathbf{a}, \mathbf{b}$, respectively. Orange arrows point to the regions of the domain walls.

a

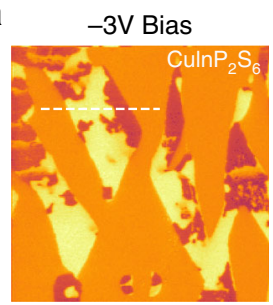

f

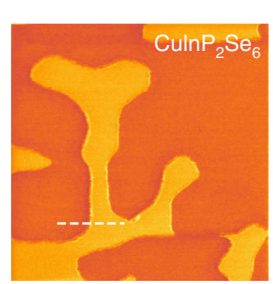

b

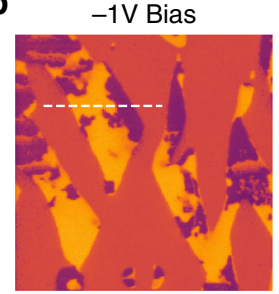

g

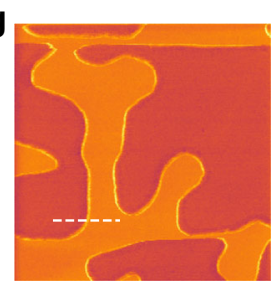

C

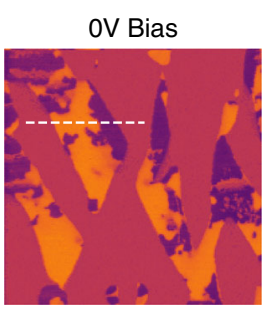

h

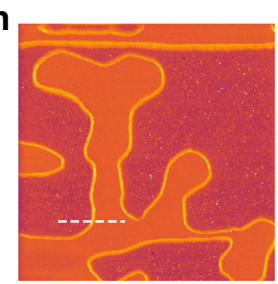

d
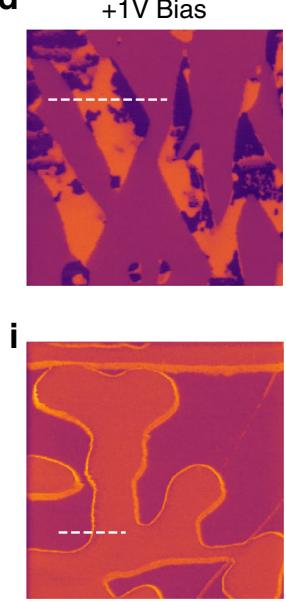

e
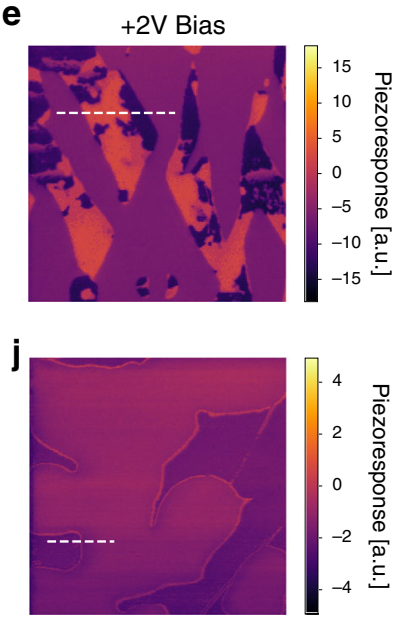

Fig. 2 Bias-dependent piezoresponse imaging of $\mathbf{C u I n}_{\mathbf{n}} \mathbf{S}_{\mathbf{6}}$ and $\mathbf{C u I n}_{\mathbf{n}} \mathbf{S}_{\mathbf{6}}$. $\mathbf{a}-\mathbf{e}$ Culn $\mathbf{P}_{2} \mathbf{S}_{6}$ imaged (image size $1.5 \mu \mathrm{m} \times 1.5 \mu \mathrm{m}$ ) at room temperature in a controlled environment and $\mathbf{f}-\mathbf{j}$ CulnP $_{2} \mathrm{Se}_{6}$ imaged (image size $2.0 \mu \mathrm{m} \times 2.0 \mu \mathrm{m}$ ) at $140 \mathrm{~K}$ in ultrahigh vacuum. Dotted white lines indicate line profile locations for Fig. 3(a,b). The bias applied to the tip is specified in the top row.

regions. Given the propensity of $\mathrm{CuInP}_{2} \mathrm{Se}_{6}$ toward antiferroelectric ordering ( ${ }^{12}$ and see below), the polar and antipolar regions would correspond to ferrielectric and antiferroelectric phases, respectively.

This scenario of phase separation can be rationalized by considering the real-space structures underpinning the hallmark property of antiferroelectrics-a double-hysteresis switching loop (Fig. 3d $)^{26-28}$. Double hysteresis corresponds to the switching of antiferroelectric configuration with net zero polarization to ferroelectric structure with nonzero polarization. Uniform antiferroelectric structure is, therefore, expected only in a very small range of applied fields ${ }^{27}$. At finite field, the system exhibits a state of finite polarization. In the real space, the structure with finite polarization can manifest as two distinct states: either the system is uniformly polarized in an applied field, or it exhibits nonuniform distribution of polarization (schematically shown in the inset of Fig. 3d), such that local regions of ferrielectric and antiferroelectric phases emerge. We believe the second case is the appropriate representation of $\mathrm{CuInP}_{2} \mathrm{Se}_{6}$ in our measurements.

Our argument for the coexistence of ferrielectric and antiferroelectric phases in $\mathrm{CuInP}_{2} \mathrm{Se}_{6}$ is supported by Raman spectroscopy and DFT calculations. Both Raman and SHG 

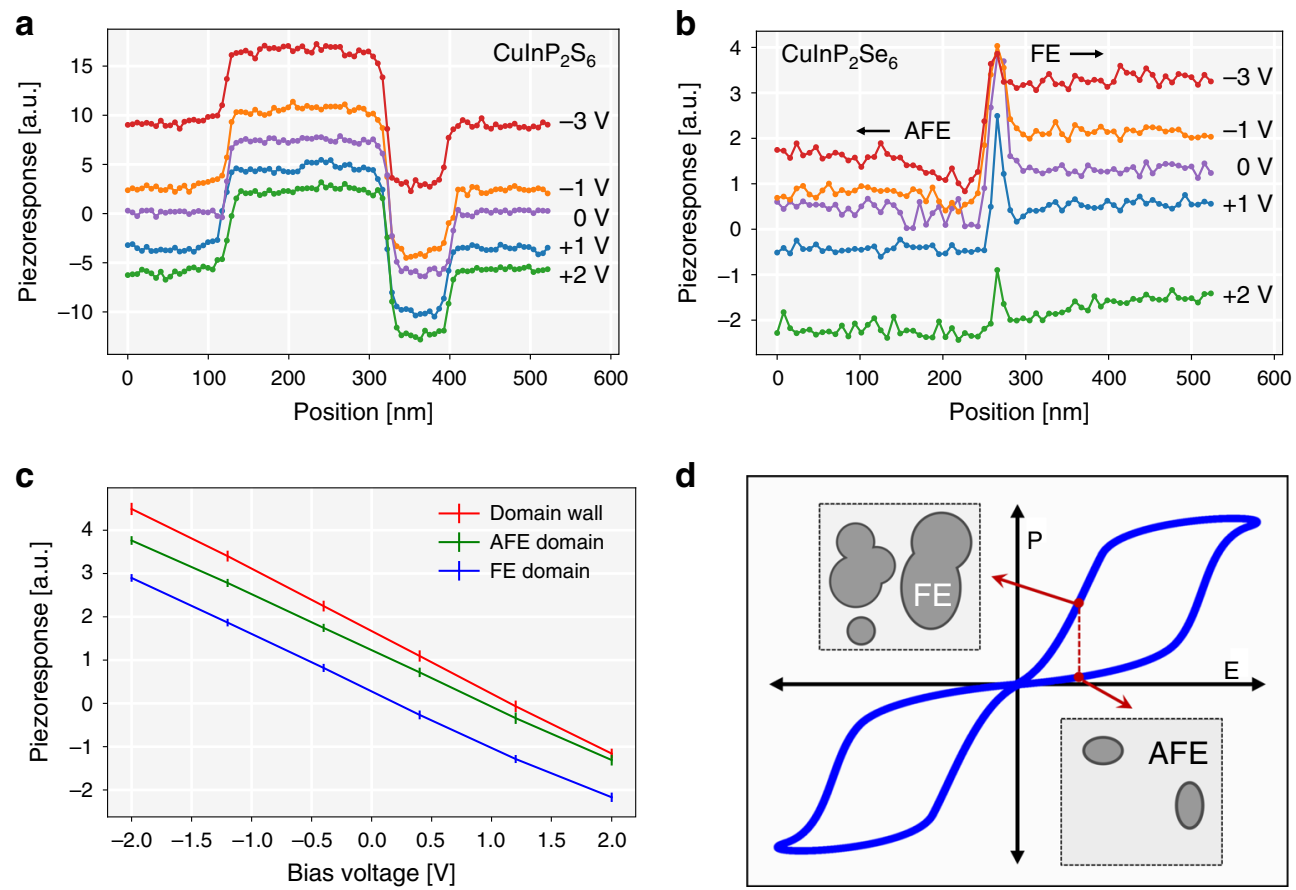

Fig. 3 Comparison of bias-dependent piezoresponse between $\mathbf{C u I n P}_{\mathbf{2}} \mathbf{S}_{\mathbf{6}}$ and $\mathbf{C u I n P}_{\mathbf{2}} \mathbf{S e}_{\mathbf{6}}$. Piezoresponse line scans presented in Fig. $2(\mathrm{a}-\mathrm{j})$ for a CulnP $\mathrm{S}_{6}$ and $\mathbf{b}$ CulnP ${ }_{2} \mathrm{Se}_{6}$ at $\mathrm{DC}$ biases ranging from $-3 \mathrm{~V}$ to $+2 \mathrm{~V}$. $\mathbf{c}$ CulnP ${ }_{2} \mathrm{Se}_{6}$ piezoresponse as a function of applied voltage for domain wall, $\mathrm{AFE}$ domain, and $\mathrm{FE}$ domain derived from Supplementary Fig. 2(d) Schematic of a canonical double-hysteresis loop for antiferroelectrics. Insets illustrate a schematic top view of the surface, with spatially separated AFE (light gray) and FE (dark gray) domains.

spectroscopy, carried out on freshly cleaved crystalline flakes of $\mathrm{CuInP}_{2} \mathrm{Se}_{6}$, clearly detect the transition below $\sim 250 \mathrm{~K}$ (Fig. 4a, d). The peaks at 203 and $219 \mathrm{~cm}^{-1}$ in Raman spectrum appear first at around $250 \mathrm{~K}$ (Fig. $4 \mathrm{a}, \mathrm{b}$ ) and their integrated intensity continues to grow, eventually saturating below $\sim 180 \mathrm{~K}$ (Supplementary Fig. 3). Simultaneously measured SHG intensity (Fig. 4d) shows a rapid increase below $220 \mathrm{~K}$. Notably, SHG detects breaking of the inversion symmetry, therefore implying the development of either ferri- or ferroelectric ordering in the probed volume of the technique.

Further, we compared the experimental Raman spectra to those calculated by DFT in the ferrielectric and antiferroelectric ground states, as shown in Fig. 4c. The AFE state in these calculations corresponds to an alternating displacement of $\mathrm{Cu}$ atoms along the b (or a) crystallographic direction, between opposite sides of each layer (Supplementary Fig. 4b), consistent with the prior work ${ }^{12}$. The $\operatorname{In}^{3+}$ in this case is located near the equatorial plane of each layer. In contrast, in the ferrielectric state, the $\mathrm{Cu}^{1+}$ and $\mathrm{In}^{3+}$ are offset in opposite directions relative to the midplane of the layers, but the offsets of each are identical within each cation sublattice.

The characteristic calculated signature of the AFE state appears to be a peak around $300 \mathrm{~cm}^{-1}$. It is negligible for the calculated spectra for the FE configurations, while prominent in the experiment (Fig. 4c). Meanwhile, the three-peak structure between $400 \mathrm{~cm}^{-1}$ and $500 \mathrm{~cm}^{-1}$ is best captured by a combination of the FE and AFE states. The probing volume of piezoresponse force microscopy measurements are comparable to the radius of the tip, $\sim 20-50 \mathrm{~nm}$, while that of optical spectroscopies $\sim 1 \mu \mathrm{m}$; thus, both are still probing near-surface regions. Note, the X-ray diffraction study of $\mathrm{CuInP}_{2} \mathrm{Se}_{6}$ across the phase transition, on the other hand, shows that the structure of

$\mathrm{CuInP} \mathrm{Se}_{6}$ belongs to the non-centrosymmetric space group P31c (No. 159) at $100 \mathrm{~K}$ and $180 \mathrm{~K}$ (Supplementary Table 1a), Supplementary Fig. 5). At $250 \mathrm{~K}$, the best fit is to the centrosymmetric space group $P \overline{3} 1 c$ (No. 163) (Supplementary
Table 1c)). These results largely agree with previous work ${ }^{8}$, indicating order-disorder type ferrielectric ordering in $\mathrm{CuInP}_{2} \mathrm{Se}_{6}$. Bulk-averaging measurements are therefore most consistent with ferrielectric ordering (Supplementary Fig. 5, Supplementary Fig. 6). However, in light of the similar energies between ferroelectric and antiferroelectric states (Supplementary Fig. 4), it is likely that uniform states of either kind can be preferred depending on specific experimental conditions.

Further insight into the structure and energetics of the AFE and FE phases and the AFE/FE domain walls has been gained using DFT calculations of large supercells, which accommodate such boundaries. The energetics and structures of several possible AFE configurations are shown in Supplementary Fig. 4. Consistent with prior work ${ }^{12}$, under zero strain, all bulk AFE structures have a higher energy than the bulk FE structure. Meanwhile, calculated energies of all three types of AFE/FE boundaries are small, within $2 \mathrm{meV} / \mathrm{nm}^{2}$ of each other, while the energy of a FE/FE domain wall is much higher, $21 \mathrm{meV} / \mathrm{nm}^{2}$ (Fig. 5). This result indicates that the formation of the observed mixed AFE/FE state is largerly governed by the energetics of the respective domains and that there is a high likelihood of formation of AFE/FE boundaries.

\section{Discussion}

The complete analysis of phase coexistence $\mathrm{CuInP}_{2} \mathrm{Se}_{6}$ falls outside the scope of this manuscript. However, we have carried out analytical modeling of the mixed free energy functional within the Landau-Ginzburg-Devonshire framework (Supplementary Discussion). The functional incorporates the contributions to the free energy from ferroelectric, antiferroelectric, antiferrodistortive order parameters, as well as coupling and striction terms. Importantly, although the coexistence of antiferroelectric and ferroelectric order parameters is intuitively simple, its detailed functional is very complicated. The variation of the order parameter across the domain wall is opposite for the ferroelectric and 
a

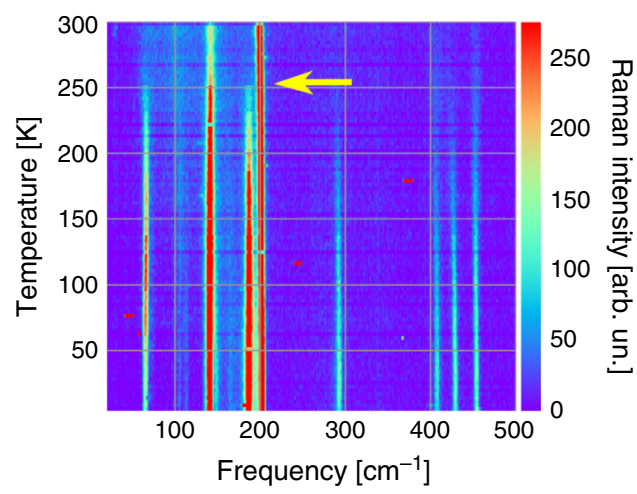

C

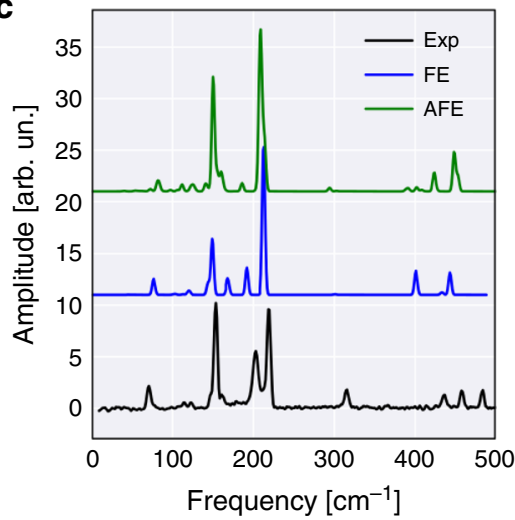

b

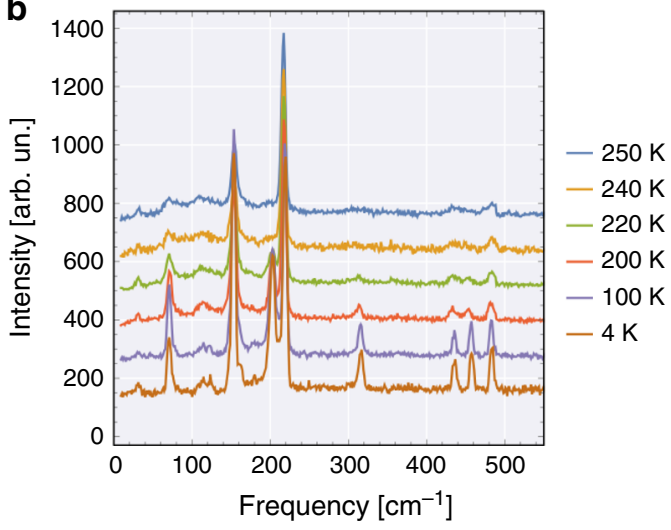

d

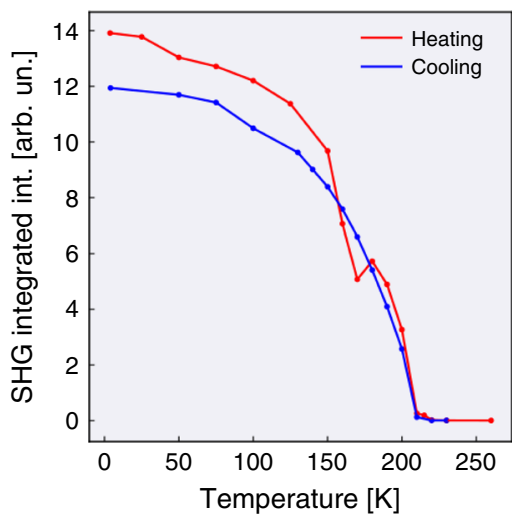

Fig. 4 Phase transition in Culn $_{\mathbf{2}} \mathbf{S e}_{\mathbf{6}}$ detected by optical spectroscopies. a Temperature dependent Raman spectroscopy of a CulnP $\mathrm{S}_{2} \mathrm{C}_{6} \mathrm{crystal}_{\text {, }}$ represented as a heat map. The phase transition below $\sim 250 \mathrm{~K}$ is clearly visible (yellow arrow). b Individual Raman spectra at several measured temperatures. c Comparison of the Raman spectrum at $7 \mathrm{~K}$ to the DFT calculations for the ferroelectric (FE) and antiferroelectric (AFE) configurations. d The intensity of second harmonic generation (SHG) across the phase transition at $200 \mathrm{~K}$.

antiferroelectric end-states: in ferroelectrics, polarization drops to zero at the domain wall ( $x=x_{0}$ in Supplementary Eq. (18)), while the antiferroelectric order parameter $\mathrm{A}$ increases at the domain wall $\left(x=y_{0}\right.$ in Supplementary Eq. (19)). Our experimental observable is strain, and specifically piezoresponse measured as a voltage derivative of strain. In the case of the FE/AFE boundary we do indeed predict a maximum strain at the wall within the approximations of the model (Supplementary Fig. 7). This behavior can be rationalized by analogy with double-hysteresis strain loops in macroscopic antiferroelectric switching (schematic in Fig. 3d). Upon onset of polarization (transition from AFE to FE phase), the lattice experiences maximum deformation (expansion in perovskite oxides ${ }^{29,30}$ ) that exceeds subsequent deformation in the ferroelectric state (due to piezoresponse). In our interpretation, the AFE-FE domain boundary in $\mathrm{CuInP}_{2} \mathrm{Se}_{6}$ is precisely the region of phase separation. The result of the LGD modeling, showing maximum strain at the boundary, supports the analogy between AFE/FE transition due to electric field (in macroscopic double-hysteresis loops) and due to phase separation in real-space (in our measurements). Although we cannot yet model the piezoresponse (dynamic strain), it is also maximized in the macroscopic hysteresis loops at the AFE/FE switching, and could therefore be maximum in our measurements in Fig. 1 and 2. Based on these results, piezoelectric domain walls should also be generally found in partially polarized antiferroelectrics.

Using quantitative piezoelectric microscopy, combined with DFT calculations and optical spectroscopy, we have revealed unusual domain-wall properties in polar $\mathrm{CuInP}_{2} \mathrm{Se}_{6}$, where domain walls exhibit maximum piezoresponse at the domain walls in contrast to the expectations for ferroelectrics. Whereas polar walls were previously detected in in antiferroelectric $\mathrm{PbZrO}_{3}{ }^{31}$ and in ferroelastic $\mathrm{CaTiO}_{3}{ }^{32,33}$, we have inferred that in $\mathrm{CuInP} \mathrm{P}_{2} \mathrm{Se}_{6}$ the domain walls separate regions of antiferroelectric and ferrielectic ordering. These domain walls should be general for antiferroelectrics while being distinct from domain walls in either ferroelectric or antiferroelectric phases, thus emerging as a new entity in the context of domain-wall electronics ${ }^{19}$. Meanwhile, the observation of antiferroelectric state confirms theoretical modeling of very small energy differences between ferrielectric and antiferroelectic states in $\mathrm{CuInP}_{2} \mathrm{Se}_{6}$. The ability to define and control locally polarized, mesoscale regions in an otherwise non-polar matrix may provide a path to integrate ferroic and electronic functionality via van der Waals interfaces, which is complementary to ferroelectric materials and that may persist down to single layer ${ }^{12}$.

\section{Methods}

Crystal growth. The single crystal $\mathrm{CuInP}_{2} \mathrm{Se}_{6}$ was grown from gas phase by chemical transport reactions. Iodine has been used as transport agent with concentration of $4-5 \mathrm{mg} / \mathrm{cm}^{3}$. The temperature of evaporation zone was $870 \mathrm{~K}$, and for the crystallization zone temperature equals $850 \mathrm{~K}$. The duration of the growth process was $350 \mathrm{~h}$. The resulting product was thin single crystal plates with dimensions near $10 \times 5 \times 0.1 \mathrm{~mm}^{3}$. $\mathrm{Cu}_{0.4} \mathrm{In}_{1.2} \mathrm{P}_{2} \mathrm{~S}_{6}$ single crystals were synthesized through the vapor transport method. Starting materials, sealed in fused silica ampules, were heated to $750-775^{\circ} \mathrm{C}$ at a rate of $30^{\circ} \mathrm{C} / \mathrm{h}$ and held at that temperature for 4 days and then cooled at a rate of $20^{\circ} \mathrm{C} / \mathrm{h}$.

PFM imaging. Ultrahigh vacuum contact PFM imaging and polarization switching were performed on an Omicron AFM/STM, interfaced with a Nanonis controller package. The chamber pressure was $1 \times 10^{-10}$ mbar or better. The samples were mounted on a standard Omicron sample plate and affixed with a silver conductive epoxy (Epo-Tek EJ2189-LV). A clean surface was prepared by Scotch tape method 
a

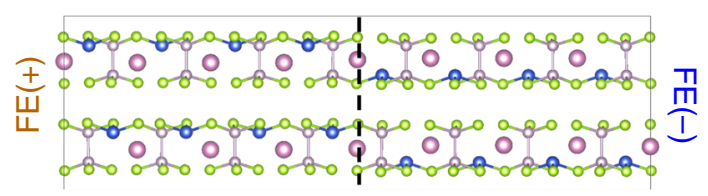

b
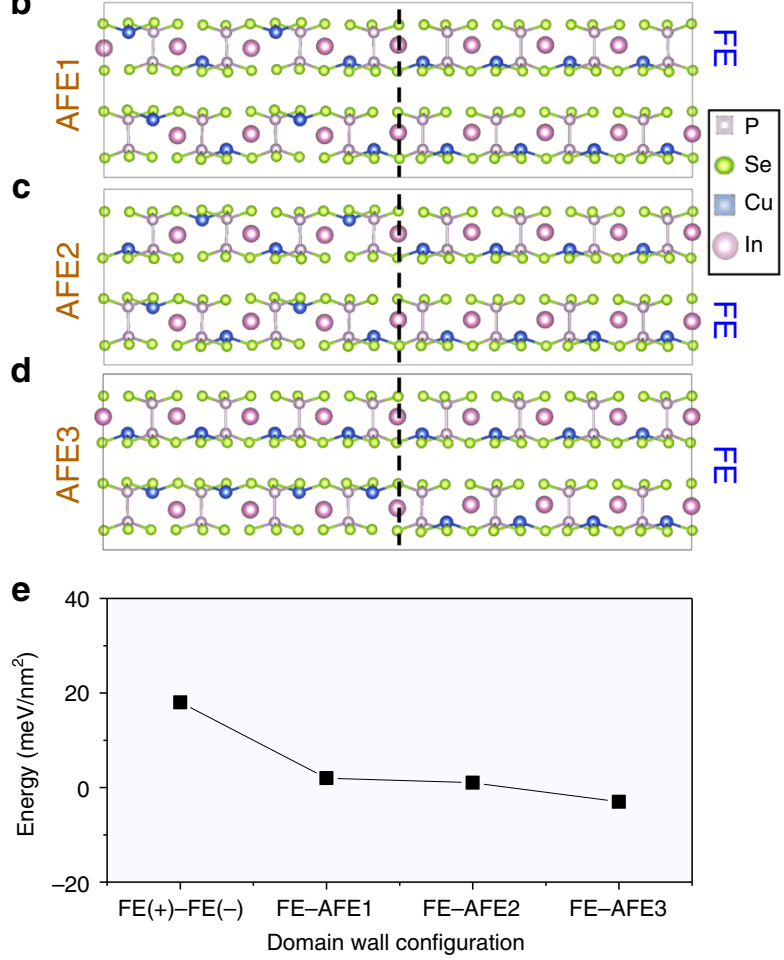

Fig. 5 Calculated structures of domain walls and phase boundaries in Culn $\mathbf{P}_{\mathbf{2}} \mathbf{S e}_{\mathbf{6}}$. a Ferrielectric domain wall (FE/FE). $\mathbf{b}$ Three kinds of antiferroelectric/ferroelectric boundaries, respectively AFE1/FE (b), AFE2/ $\mathrm{FE}$ (c) and AFE3/FE (d). e Corresponding boundary and wall energies. FE stands for ferrielectric and AFE for antiferroelectric order.

in UHV. Images of $\mathrm{CuInP}_{2} \mathrm{~S}_{6}$ shown in this paper were acquired in an Ar-filled glove-box using Bruker-Icon AFM. The freshly cleaved surface was likewise prepared shortly before the measurement.

Single crystal XRD. The structure of single crystals was characterized by using a Rigaku XtalLAB PRO diffractometer with graphite monochromatized Mok-alpha radiation (lambda $=0.71073$ Angstrom) equipped with a Dectris Pilatus $200 \mathrm{~K}$ and an Oxford Cryosystems $\mathrm{N}$-HeliX cryocooler for temperature ranging from 100 to $293 \mathrm{~K}$.

Raman spectroscopy. The Raman spectra were measured in a custom-built micro-Raman setup. The samples were excited with a continuous wave (cw) diodepumped solid-state laser (Excelsior, Spectra Physics, $532 \mathrm{~nm}, 100 \mathrm{~mW}$ ) through an upright microscope using a $\times 50$ long-working distance objective with NA (numeric aperture) $=0.5$. The typical incident laser power on a sample was maintained at $\sim$ $100 \mu \mathrm{W}$ to reduce possible laser heating and damaging of the samples during Raman spectra acquisition. The scattered Raman light was analyzed by a spec trometer (Spectra Pro 2300i, Acton, $f=0.3 \mathrm{~m}$ ) that was coupled to the microscope and equipped with a 1800 groves/mm grating and a CCD camera (Pixis 256BR, Princeton Instruments). The low-temperature Raman spectra were measured using a liquid He-cryostat (MicrostatHiResII, Oxford Instruments) with a temperature controller (MercuryiT, Oxford Instruments) that allowed precise control from 3.6 to $300 \mathrm{~K}$. Raman spectra were sampled with $10 \mathrm{~K}$ step from 100 to $250 \mathrm{~K}$, and also at 4,7 , and $20 \mathrm{~K}$. The cryostat was mounted on a motorized $X Y$ microscope stage (Marzhauser) under the microscope of the micro-Raman setup. The cryostat was evacuated to the base pressure of $7 \times 10^{-7} \mathrm{mbar}$ prior to cool down

Second harmonic measurements. Second harmonic generation (SHG) measurements were conducted using a $50 \mathrm{fs}$ Ti:sapphire laser (Micra, Coherent) at 800 $\mathrm{nm}$ and $80 \mathrm{MHz}$ repetition rate. The laser beam was passed through a half-wave plate mounted in a rotation stage and was directed into an upright microscope (Olympus) and focused onto a sample surface using a $\times 50$ microscope objective
(Numerical Aperture: $\mathrm{NA}=0.5$ ) to a few micron spot. The laser energy at the sample surface was $\sim 0.1 \mathrm{~W}$. The SHG light was collected in backscattering configuration using the same objective and was directed to a monochromator (Spectra Pro 2300i, Acton, $f=0.3 \mathrm{~m}$ ) that was coupled to the microscope and equipped with a 150 grooves $/ \mathrm{mm}$ grating and a CCD camera (Pixis 256BR, Princeton Instruments). Before entering the monochromator, the SHG light was passed through a short-pass cutoff filter $(650 \mathrm{~nm})$ and a polarizer to filter out the fundamental excitation light at $800 \mathrm{~nm}$ and select the SHG polarization parallel to that of the excitation light. The low-temperature SHG measurements were conducted using the same liquid He-cryostat, which was used for Raman measurements.

DFT calculations. The DFT calculations (relaxations and $\Gamma$-point phonon frequencies) in this study use the VASP 5.3.5 computational package ${ }^{34}$ and are carried out under the Perdew-Burke-Ehrenhof generalized gradient approximation (GGA) and the the DFT-D2 method as developed by Grimme ${ }^{35}$. The recommended VASP PAW pseudopotentials were used. All calculations used a $600 \mathrm{eV}$ energy cutoff. Raman frequencies and intensities are calculated using the package developed by Fonari and Stauffer ${ }^{36}$. The calculations were performed on two phases of $\mathrm{CuInP}_{2} \mathrm{Se}_{6}$ : a 20 -atom ferroelectric $(\mathrm{FE})$ phase, and a 40 -atom $1 \times 2 \times 1$ antiferrolectric (AFE) phase. The setup for the $\mathrm{Cu}$ atoms in the AFE unit cell is the same as that found by Song et al. ${ }^{12}$ For phonon calculations, both structures were relaxed so that the forces are less than $5 \mathrm{e}^{-8} \mathrm{eV} / \AA$ to eliminate residual spurious forces. The FE/FE and FE/AFE domain-wall calculations are performed using $8 \times$ $2 \times 1$ supercells containing 160 atoms. These calculations use a $\Gamma$-centered Monkhorst-Pack (MP) k-point grid of $1 \times 4 \times 2$. All atoms were relaxed until all forces were smaller than $0.02 \mathrm{eV} / \AA$. The domain boundary energy per unit area is calculated as

$$
E_{\text {boundary }}=\left(E_{\text {total }}-E_{\text {phase }_{2}}\right) / 2 S \text {, }
$$

where $E_{\text {total }}$ is the supercell energy, $E_{\text {phase1 }}$ and $E_{\text {phase2 }}$ are the energies for the requisite number of atoms in those phases, and $S$ is the cross-sectional area of the boundary.

\section{Data availability}

Source data for the scanning probe images, results of optical spectroscopy and first principles calculations presented in this manuscript will be shared upon reasonable request.

\section{Code availability}

The data analysis was carried out with widely accepted commercial and open source software without any significant code customization.

Received: 6 September 2019; Accepted: 6 June 2020; Published online: 17 July 2020

\section{References}

1. Susner, M. A., Chyasnavichyus, M., McGuire, M. A., Ganesh, P. \& Maksymovych, P. Metal thio- and selenophosphates as multifunctional van der Waals layered materials. Adv. Mater. 29, 1602852 (2017).

2. Chang, K. et al. 2D ferroelectrics: enhanced spontaneous polarization in ultrathin SnTe films with layered antipolar structure. Adv. Mater. 31, 1970016 (2019)

3. Wu, M. \& Jena, P. The rise of two-dimensional van der Waals ferroelectrics Wiley Interdiscip. Rev.: Comput. Mol. Sci. 8, e1365 (2018).

4. Chang, K. et al. Discovery of robust in-plane ferroelectricity in atomic-thick SnTe. Science 353, 274-278 (2016).

5. Liu, F. et al. Room-temperature ferroelectricity in $\mathrm{CuInP}_{2} \mathrm{~S}_{6}$ ultrathin flakes. Nat. Commun. 7, 12357 (2016)

6. Maisonneuve, V., Cajipe, V. B., Simon, A., Von Der Muhll, R. \& Ravez, J. Ferrielectric ordering in lamellar CuInP ${ }_{2} \mathrm{~S}_{6}$. Phys. Rev. B 56, 10860-10868 (1997).

7. Simon, A., Ravez, J., Maisonneuve, V., Payen, C. \& Cajipe, V. B. Paraelectric-ferroelectric transition in the lamellar thiophosphate $\mathrm{CuInP}_{2} \mathrm{~S}_{6}$. Chem. Mater. 6, 1575-1580 (1994).

8. Bourdon, X., Maisonneuve, V., Cajipe, V. B., Payen, C. \& Fischer, J. E. Copper sublattice ordering in layered $\mathrm{CuMP}_{2} \mathrm{Se}_{6}(\mathrm{M}=\mathrm{In}, \mathrm{Cr})$. J. Alloy. Compd. 283, 122-127 (1999).

9. Vysochanskii, Yu. M., Molnar, A. A., Gurzan, M. I., Cajipe, V. B. \& Bourdon, $\mathrm{X}$. Dielectric measurement study of lamellar $\mathrm{CuInP}_{2} \mathrm{Se}_{6}$ : successive transitions towards a ferroelectric state via an incommensurate phase? Solid State Commun. 115, 13-17 (2000).

10. Liubachko, V. et al. Anisotropic thermal properties and ferroelectric phase transitions in layered $\mathrm{CuInP}_{2} \mathrm{~S}_{6}$ and $\mathrm{CuInP}_{2} \mathrm{Se}_{6}$ crystals. J. Phys. Chem. Solids 111, 324-327 (2017).

11. Brehm, J. A. et al. Tunable quadruple-well ferroelectric van der Waals crystals. Nat. Mater. 19, 43-48 (2020). 
12. Song, W., Fei, R. \& Yang, L. Off-plane polarization ordering in metal chalcogen diphosphates from bulk to monolayer. Phys. Rev. B 96, 235420 (2017).

13. Vysochanskii, Y. M., Molnar, A. A., Gurzan, M. I. \& Cajipe, V. B. Phase transitions in $\mathrm{CuInP}_{2}\left(\mathrm{Se}_{x} \mathrm{~S} 1-x\right)_{6}$ layered crystals. Ferroelectrics 257, 147-154 (2001).

14. Junquera, J. \& Ghosez, P. Critical thickness for ferroelectricity in perovskite ultrathin films. Nature 422, 506-509 (2003).

15. Belianinov, A. et al. $\mathrm{CuInP}_{2} \mathrm{~S}_{6}$ room temperature layered ferroelectric. Nano Lett. 15, 3808-3814 (2015)

16. Chyasnavichyus, M. et al. Size-effect in layered ferrielectric $\mathrm{CuInP}_{2} \mathrm{~S}_{6}$. Appl. Phys. Lett. 109, 172901 (2016).

17. Neumayer, S. M. et al. Giant negative electrostriction and dielectric tunability in a van der Waals layered ferroelectric. Phys. Rev. Mater. 3, 24401 (2019).

18. Meier, D. Functional domain walls in multiferroics. J. Phys. Condens. Matter 27, 463003 (2015).

19. Catalan, G., Seidel, J., Ramesh, R. \& Scott, J. F. Domain wall nanoelectronics. Rev. Mod. Phys. 84, 119-156 (2012).

20. Rodriguez, B. J. et al. Domain growth kinetics in lithium niobate single crystals studied by piezoresponse force microscopy. Appl. Phys. Lett. 86, 012906 (2005).

21. Kalinin, S. V., Rar, A. \& Jesse, S. A decade of piezoresponse force microscopy: progress, challenges, and opportunities. IEEE Trans. Ultrason. Ferroelectr. 53, 2226-2252 (2006).

22. Jesse, S. \& Kalinin, S. V. Band excitation in scanning probe microscopy: sines of change. J. Phys. D. Appl. Phys. 44, 464006 (2011).

23. Jesse, S. et al. Resolution theory, and static and frequency-dependent crosstalk in piezoresponse force microscopy. Nanotechnology 21, 405703 (2010).

24. Balke, N. et al. Exploring local electrostatic effects with scanning probe microscopy: implications for piezoresponse force microscopy and triboelectricity. ACS Nano 8, 10229-10236 (2014).

25. Jesse, S., Mirman, B. \& Kalinin, S. V. Resonance enhancement in piezoresponse force microscopy: Mapping electromechanical activity, contact stiffness, and Q factor. Appl. Phys. Lett. 89, 022906 (2006).

26. Hatt, R. A. \& Cao, W. Landau-Ginzburg model for antiferroelectric phase transitions based on microscopic symmetry. Phys. Rev. B 62, 818-823 (2000).

27. Tolédano, P. \& Guennou, M. Theory of antiferroelectric phase transitions. Phys. Rev. B 94, 014107 (2016).

28. Misirlioglu, I. B., Pintilie, L., Boldyreva, K., Alexe, M. \& Hesse, D. Antiferroelectric hysteresis loops with two exchange constants using the two dimensional Ising model. Appl. Phys. Lett. 91, 202905 (2007).

29. Pan, W., Zhang, Q., Bhalla, A. \& Cross, L. E. Field-forced antiferroelectric-toferroelectric switching in modified lead zirconate titanate stannate ceramics. J. Am. Ceram. Soc. 72, 571-578 (1989).

30. Zhou, L., Zimmermann, A., Zeng, Y.-P. \& Aldinger, F. Fatigue of field-induced strain in antiferroelectric $\mathrm{Pb}_{0.97} \mathrm{La}_{0.02}\left(\mathrm{Zr}_{0.77} \mathrm{Sn}_{0.14} \mathrm{Ti}_{0.09}\right) \mathrm{O}_{3}$ Ceramics. J. Am. Ceram. Soc. 87, 1591-1593 (2004).

31. Wei, X.-K. et al. Ferroelectric translational antiphase boundaries in nonpolar materials. Nat. Commun. 5, 3031 (2014).

32. Barone, P., Di Sante, D. \& Picozzi, S. Improper origin of polar displacements at $\mathrm{CaTiO}_{3}$ and $\mathrm{CaMnO}_{3}$ twin walls. Phys. Rev. B 89, 144104 (2014).

33. Aert, S. V. et al. Direct observation of ferrielectricity at ferroelastic domain boundaries in $\mathrm{CaTiO}_{3}$ by electron microscopy. Adv. Mater. 24, 523-527 (2012).

34. Kresse, G. \& Furthmüller, J. Efficient iterative schemes for ab-initio totalenergy calculations using a plane-wave basis set. Phys. Rev. B 54, 11169-11186 (1996).

35. Grimme, S. Semiempirical GGA-type density functional constructed with a long-range dispersion correction. J. Comput. Chem. 27, 1787-1799 (2006).

36. Fonari, A. \& Stauffer, S. vasp_raman.py. https://github.com/raman-sc/VASP/ (2013).

\section{Acknowledgements}

A portion of the work at ORNL, including ambient scanning probe microscopy and data interpretation, were supported by the US Department of Energy, Office of Science, Basic Energy Sciences, Materials Science and Engineering Division. A.D. wish to acknowl- edgement the support of the CIEE/Baltic-American Freedom Foundation in funding parts of this work. Raman, SHG, and SPM measurements were conducted at the Center for Nanophase Materials Sciences, which is a DOE Office of Science User Facility. A.N. M. acknowledges National Academy of Sciences of Ukraine and has received funding from the European Union's Horizon 2020 research and innovation program under the Marie Skłodowska-Curie grant agreement No 778070. The work at Vanderbilt University was supported in part by the U.S. Department of Energy, Office of Science, Basic Energy Sciences, Materials Science and Engineering Division grant DE-FG02-09ER46554 and by the McMinn Endowment at Vanderbilt University. Calculations were performed at the National Energy Research Scientific Computing Center, a DOE Office of Science User Facility supported by the Office of Science of the U.S. Department of Energy under Contract No. DE-AC02-05CH11231 and using systems provided by the U.S. Department of Defense's High Performance Computing Modernization Program (HPCMP). The authors are grateful for funding support from AFOSR (LRIR\#16RXCOR322)

\section{Author contributions}

A.D., K.K., S.N., M.C. carried out imaging experiments in ambient and vacuum environments. A.P. carried out Raman and SHG spectroscopies. F.Y., B.C. carried out X-ray diffraction. J.A.B., L.T., A.O. carried out first principles modeling of structure and domain walls. S.T.P. supervised the DFT calculations and participated in theoretical analysis and paper writing. T.F. and J.A.B. calculated Raman spectra. M.A.S., M.A.M. provided the thiophosphate crystal, helped with data interpretation and manuscript writing. E.A.E. and A.N.M. carried out analytical modeling. A.D.,. J.B., Y.V. provided the selenide crystal. A.D., J.B., Y.V., N.B., S.V.K. helped with data interpretation and manuscript writing. P.G. helped with theoretical interpretation and manuscript writing P.M. led the research effort and manuscript writing. P.M., K.K., and A.N.M. developed the ferroelectric/antiferroelectric phase coexistence picture.

\section{Competing interests}

The authors declare no competing interests.

\section{Additional information}

Supplementary information is available for this paper at https://doi.org/10.1038/s41467020-17137-0.

Correspondence and requests for materials should be addressed to P.M.

Peer review information Nature Communications thanks Chun-Gang Duan, Dmitri Tenne and the other, anonymous, reviewer(s) for their contribution to the peer review of this work.

Reprints and permission information is available at http://www.nature.com/reprints

Publisher's note Springer Nature remains neutral with regard to jurisdictional claims in published maps and institutional affiliations.

Open Access This article is licensed under a Creative Commons Attribution 4.0 International License, which permits use, sharing, adaptation, distribution and reproduction in any medium or format, as long as you give appropriate credit to the original author(s) and the source, provide a link to the Creative Commons license, and indicate if changes were made. The images or other third party material in this article are included in the article's Creative Commons license, unless indicated otherwise in a credit line to the material. If material is not included in the article's Creative Commons license and your intended use is not permitted by statutory regulation or exceeds the permitted use, you will need to obtain permission directly from the copyright holder. To view a copy of this license, visit http://creativecommons.org/ licenses/by/4.0/

This is a U.S. Government work and not under copyright protection in the US; foreign copyright protection may apply 2020 